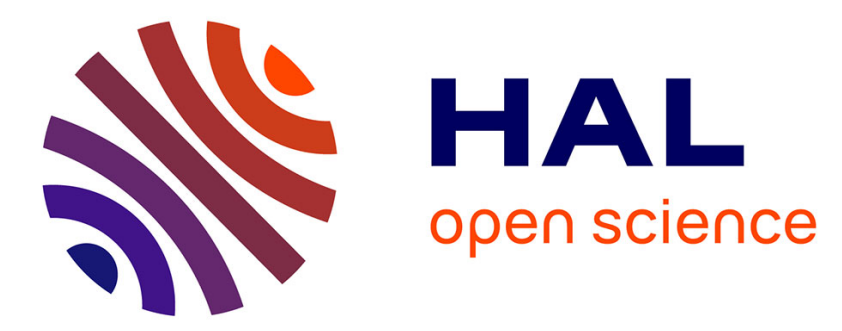

\title{
The depolarized Raman 2 nu(3) overtone of CO2: A line-mixing shape analysis
} I.-A. Verzhbitskiy, A.-P. Kouzov, Florent Rachet, Michel Chrysos

\section{To cite this version:}

I.-A. Verzhbitskiy, A.-P. Kouzov, Florent Rachet, Michel Chrysos. The depolarized Raman 2 nu(3) overtone of CO2: A line-mixing shape analysis. Journal of Chemical Physics, 2011, 134 (19), Non spécifié. 10.1063/1.3580278. hal-03344693

\section{HAL Id: hal-03344693 \\ https://univ-angers.hal.science/hal-03344693}

Submitted on 15 Sep 2021

HAL is a multi-disciplinary open access archive for the deposit and dissemination of scientific research documents, whether they are published or not. The documents may come from teaching and research institutions in France or abroad, or from public or private research centers.
L'archive ouverte pluridisciplinaire HAL, est destinée au dépôt et à la diffusion de documents scientifiques de niveau recherche, publiés ou non, émanant des établissements d'enseignement et de recherche français ou étrangers, des laboratoires publics ou privés. 


\section{AD| $\begin{aligned} & \text { The Journal of } \\ & \text { Chemical Physics }\end{aligned}$}

\section{The depolarized Raman 2v3 overtone of $\mathrm{CO} 2$ : A line-mixing shape analysis}

I. A. Verzhbitskiy, A. P. Kouzov, F. Rachet, and M. Chrysos

Citation: The Journal of Chemical Physics 134, 194305 (2011); doi: 10.1063/1.3580278

View online: http://dx.doi.org/10.1063/1.3580278

View Table of Contents: http://scitation.aip.org/content/aip/journal/jcp/134/19?ver=pdfcov

Published by the AIP Publishing

\section{Articles you may be interested in}

CO2 isolated line shapes by classical molecular dynamics simulations: Influence of the intermolecular potential and comparison with new measurements

J. Chem. Phys. 140, 084308 (2014); 10.1063/1.4866449

Molecular dynamics simulations for $\mathrm{CO} 2$ spectra. IV. Collisional line-mixing in infrared and Raman bands J. Chem. Phys. 138, 244310 (2013); 10.1063/1.4811518

The isotropic spectrum of the CO2 Raman 2v3 overtone: A line-mixing band shape analysis at pressures up to several tens of atmospheres

J. Chem. Phys. 134, 224301 (2011); 10.1063/1.3596750

The isotropic remnant of the CO2 near-fully depolarized Raman 2v3 overtone

J. Chem. Phys. 134, 104310 (2011); 10.1063/1.3557820

Are asymmetric stretch Raman spectra by centrosymmetric molecules depolarized?: The $2 \mathrm{v} 3$ overtone of $\mathrm{CO} 2$ J. Chem. Phys. 134, 044318 (2011); 10.1063/1.3535599

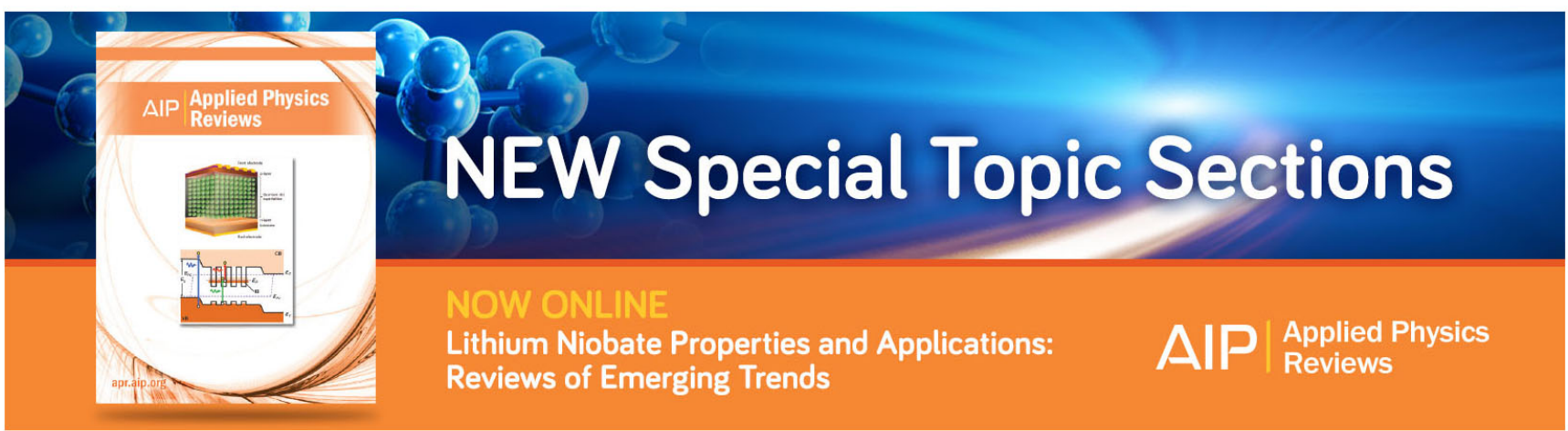




\title{
The depolarized Raman $2 v_{3}$ overtone of $\mathrm{CO}_{2}$ : A line-mixing shape analysis
}

\author{
I. A. Verzhbitskiy, ${ }^{1}$ A. P. Kouzov ${ }^{2}$ F. Rachet, ${ }^{1}$ and M. Chrysos ${ }^{1, a)}$ \\ ${ }^{1}$ MolTech-Anjou, UMR CNRS 6200, Université d'Angers, 2 Boulevard Lavoisier, 49045 Angers, France \\ ${ }^{2}$ Institute of Physics, Saint Petersburg State University, Ulyanovskaya Str. 1, Peterhof, Saint Petersburg \\ 198504, Russia
}

(Received 4 February 2011; accepted 29 March 2011; published online 18 May 2011)

\begin{abstract}
In a recent article we showed that the $2 \nu_{3}$ transition of $\mathrm{CO}_{2}$ gives rise to a Raman spectrum that is almost entirely depolarized [M. Chrysos, I. A. Verzhbitskiy, F. Rachet, and A. P. Kouzov, J. Chem. Phys. 134, 044318 (2011)]. In the present article, we go further forward in the study of this overtone by reporting a first-principles shape analysis of its depolarized spectrum at room temperature. As a first step in our analysis, a model assuming isolated Lorentzian line shapes was applied, which at low gas densities turns out to be sufficient for qualitative conclusions. As the next step, a sophisticated approach was developed on the basis of the extended strong-collision model in order to properly account for the heavy line mixing between rotational lines. Whereas a marked deviation between model and measured spectra was observed upon application of the simpler model, striking agreement even at the highest $\mathrm{CO}_{2}$ density was found on applying the sophisticated one. Accurate calculated data were used for the rotational line broadening coefficients without resort to arbitrary parameters. Values for the vibrational shift scaling linearly with the density of the gas are given. (0) 2011 American Institute of Physics. [doi:10.1063/1.3580278]
\end{abstract}

\section{INTRODUCTION}

Spectral lines in gases are merely the result of the interactions between the gas molecules and an applied electromagnetic field. Nonetheless, except at very low gas pressures, the line shapes and their modeling can be greatly affected by the collisions of gas particles. Efforts to develop models for line shapes date back to Anderson's seminal paper. ${ }^{1}$ Since then, numerous emblematic works have appeared in an attempt to interpret, describe and model molecular interactions. Among such, the works by Baranger and co-workers ${ }^{2-6}$ are only a few representative samples. In all those works, different approaches have been developed to mimic and interpret collisionally broadened line or band shapes. To record the profiles various spectral techniques are being employed, among which is linear or nonlinear Raman scattering spectroscopy.

During the last decades Raman scattering has been the subject of numerous experimental and theoretical investigations. These have helped scientists beef up spectroscopic databases for a variety of electro-optical properties in gases and mixtures. Such data serve a wide range of industrial applications and can play an important role in planetary and interstellar physics. Carbon dioxide is the most potent greenhouse gas in the atmosphere, and its spectroscopy has been attracting a great deal of attention over the last few decades. ${ }^{7-12}$ However, the spectral region in which the $2 v_{3}$ transition of $\mathrm{CO}_{2}$ appears has never been the subject of line shape analysis so far. The extremely weak intensity of this transitionmillions of times smaller than the intensity of a typical $v_{1}$ transition-makes the $2 v_{3}$ overtone band an appealing benchmark for line shape models. The rich threefold branch

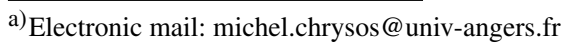

structure of its anisotropic Raman spectrum stands as an excellent representative for band shape studies.

One of the principal characteristics of MolTechAnjou Institute's incoherent Raman equipment is its high sensitivity - a property intended primarily to allow for measurements of very weak collision-induced light scattering signals. (An overview of the collision-induced light scattering processes can be found in Refs. 13 and 14.) In this respect, the collision-induced simultaneous transition that we reported recently in a series of papers ${ }^{15}$ is probably the weakest signal ever detected by our experiment and, to the best of our knowledge, a Raman signal of unprecedented feebleness. To give the reader a rough idea of what "weak" means, the aforementioned signal (in counts per second) was about one billion times weaker than that of a typical $v_{1}$ Raman transition, the latter having served as a reference for comparisons.

It is obvious from above that this performance is higher than what was necessary for the reliable detection of the $2 v_{3}$ overtone of $\mathrm{CO}_{2}$. In a preceding paper, ${ }^{16}$ the $2 v_{3}$ band was readily measured for a wide range of $\mathrm{CO}_{2}$ pressures, which permitted to deduce highly accurate values for certain electro-optical properties of $\mathrm{CO}_{2}$. The band turned out to be $98 \%$ depolarized, so its spectrum was almost exclusively anisotropic. ${ }^{16}$

Here, we present an exhaustive shape analysis of the anisotropic spectrum of the overtone, at a range of $\mathrm{CO}_{2}$ densities varying from 5 to 55 amagat. To this end, two models of quite different degrees of sophistication were employed. In the first, isolated Lorentzians were used. Albeit simple, this model was sufficient for rough predictions, especially at low gas pressures, but it was insufficient for refined quantitative analyses. The next step was to employ the "extended strong-collision model" (ESCM) (Refs. 17 and 18) - a device intended to consistently reproduce strong line 
mixing effects. It is precisely these effects that render the first model insufficient at high gas pressures. We show below that, while the deviation between the former model and the experiment is dramatically enhanced with the gas density, the response of the ESCM remains excellent over the whole density range. For rotational broadening, reliably calculated data were used, ${ }^{19}$ so we need not resort to fitting parameters. For vibrational broadening and shift, an adjustable complex constant, $\gamma_{v}+i \delta_{v}$, that was common to all the rotational lines was introduced in the diagonal of the relaxation matrix, with the purpose to determine $\gamma_{v}$ and $\delta_{v}$ through adjustment of the model band shapes to the experimental ones.

Our findings suggest that the ESCM is the most workable compromise between simplicity and accuracy in the effort to model strong line mixing effects.

\section{EXPERIMENT}

The $2 v_{3}$ band was detected in the Stokes side of the spectrum, in a frequency region that is near twice the frequency of the asymmetrical stretching fundamental vibration. The band was centered at the wave number $4673 \mathrm{~cm}^{-1}$ and recorded over a range varying from 4515 to $4810 \mathrm{~cm}^{-1}$. In order to cover properly such a wide domain, the 4515-4810 interval was divided into four separated, partially overlapping intervals. The green line $\left(\lambda_{L}=514.5 \mathrm{~nm}\right)$ of a $2 \mathrm{~W}$-power $\mathrm{Ar}^{+}$ laser was used to excite the transition $00^{0} 2 \leftarrow 00^{\circ} 0$. The experiment was carried out with a $90^{\circ}$ geometry setup. A double monochromator and a nitrogen-cooled CCD chain were employed for analysis and detection. Owing to the instrumental function, signal distortions were produced. These effects were taken into account in our calculations by means of a neartriangular $0.7 \mathrm{~cm}^{-1}$ FWHM slit. The exhaustive description of the experimental setup can be found in Ref. 20. The gas was kept at a constant temperature $(294.5 \mathrm{~K})$ with the density covering the range 5-55 amagat. The upper gas density bound was limited by the liquefaction properties of $\mathrm{CO}_{2}$.

In agreement with Atkins' general assertion "vibrations that are not totally symmetrical give rise to depolarized lines, ${ }^{21}$ our recent study showed ${ }^{16}$ that the spectrum of $2 v_{3}$ is almost entirely depolarized. The depolarized spectrum, which was by far the dominant spectral component of the overtone, was extracted from two independent signal recordings, $S_{\|}$and $S_{\perp}$, depending on whether the electric field of the incident beam was polarized in the direction parallel or perpendicular to the scattering plane.

The integrated intensity of the depolarized spectrum was found to scale with the gas density strictly linearly. This property is evidence that the observed spectrum comes from the interactions of photons with isolated $\mathrm{CO}_{2}$ molecules rather than with clusters of molecules, which would otherwise have altered the scaling law. As a consequence of this property, a line shape model that focuses on the intrinsic $\mathrm{CO}_{2}$ polarizability only and that neglects any transient polarizabilities that are induced during collisions between the radiator and the perturber in a molecular ensemble, is fully justified.

\section{ALLOWED RAMAN LINES}

\section{A. The frequency positions}

The positions of the roto-vibrational lines, in the frequency domain, are given from the following equation: ${ }^{22}$

$$
\begin{aligned}
v_{J}= & v_{00}+\left[B^{\prime} J^{\prime}\left(J^{\prime}+1\right)-B J(J+1)\right] \\
& -\left\{D^{\prime}\left[J^{\prime}\left(J^{\prime}+1\right)\right]^{2}-D[J(J+1)]^{2}\right\} \\
& +\left\{H^{\prime}\left[J^{\prime}\left(J^{\prime}+1\right)\right]^{3}-H[J(J+1)]^{3}\right\} .
\end{aligned}
$$

Here, $v_{00}$ denotes the frequency $v_{J J^{\prime}}$ of the transition $\left(v^{\prime}, J^{\prime}\right.$ $=0) \leftarrow(v, J=0)$ and $J\left(J^{\prime}\right)$ the rotational quantum number of the initial (final) state. The symbol $v\left(v^{\prime}\right)$ is an abbreviation for the sequence $v_{1} v_{2}^{l} v_{3}\left(v_{1}^{\prime} v_{2}^{\prime l^{\prime}} v_{3}^{\prime}\right)$ of the initial (final) state vibrational quantum numbers. The two sets of parameters $B, D, H$ and $B^{\prime}, D^{\prime}, H^{\prime}$ refer to spectroscopic constants for the initial and the final vibrational states, respectively. In either set, $B\left(B^{\prime}\right)$ is the leading order parameter; it is directly related to the molecule's moment of inertia, $I\left(I^{\prime}\right)$, through the expression $B=\hbar^{2} / 2 I\left(B^{\prime}=\hbar^{2} / 2 I^{\prime}\right)$. The constants $D$ and $H$ appear because of centrifugal distortion of the bond distances. In a description that goes beyond the rigid rotor approximation, this distortion cannot be neglected. For $\mathrm{CO}_{2}$, the parameter values for $B, D$, and $H$, as well as for $B^{\prime}, D^{\prime}$, and $H^{\prime}$ were deduced from accurate near-infrared high-resolution absorption measurements. ${ }^{23}$ For the transition $00^{0} 2 \leftarrow 00^{0} 0$, the values of the spectroscopic constants are gathered in Table I.

\section{B. The strengths}

The intensity of the anisotropic Raman spectrum for a transition between an initial state $(v ; J)$ and a final state $\left(v^{\prime} ; J^{\prime}\right)$ is directly proportional to the probability $P$ that this transition occurs. This probability reads

$$
P=b_{J}^{J^{\prime}}\left|\left\langle\Psi_{v, J}|\beta| \Psi_{v^{\prime}, J^{\prime}}\right\rangle\right|^{2} .
$$

Here, $\beta$ is the dipole polarizability anisotropy of $\mathrm{CO}_{2}$ and $b_{J}^{J^{\prime}}$ denotes (squared) Clebsh-Gordan coefficients. There are three possibilities that are allowed for $b_{J}^{J^{\prime}}\left(J^{\prime}=J, J \pm 2\right)$, so the anisotropic spectrum consists of three branches. Depending on whether $\Delta J\left(=J^{\prime}-J\right)=-2$, 0 , or 2 , the three branches are identified as $O, Q$, or $S$, respectively. The rotation-vibration couplings are introduced in this description through the matrix element $\left\langle\Psi_{v, J}|\beta| \Psi_{v^{\prime}, J^{\prime}}\right\rangle$. As was borne out from our analysis, in the case of the $2 \nu_{3}$ band of $\mathrm{CO}_{2}$, these couplings are so weak that they should simply be ignored for

TABLE I. Spectroscopic constants for the $00^{0} 2 \leftarrow 00^{0} 0$ overtone transition. All quantities are in $\mathrm{cm}^{-1}$.

\begin{tabular}{lcc}
\hline \hline$\nu_{00}$ & $B$ & $B^{\prime}$ \\
\hline $4673.3253699(112)$ & $0.390218949(36)$ & $0.384066147(1)$ \\
& $D \times 10^{6}$ & $D^{\prime} \times 10^{6}$ \\
& $0.1334088(186)$ & $0.13270177(96)$ \\
& $H \times 10^{12}$ & $H^{\prime} \times 10^{12}$ \\
& $0.01918(250)$ & $0.015008(214)$ \\
\hline \hline
\end{tabular}


the purposes of this study. The paragraph below outlines this issue.

According to Herman and Wallis, ${ }^{24}$ the quantity $\left|\left\langle\Psi_{v, J}|\beta| \Psi_{v^{\prime}, J^{\prime}}\right\rangle\right|^{2}$ can be expressed as

$$
\left|\left\langle\Psi_{v, J}|\beta| \Psi_{v^{\prime}, J^{\prime}}\right\rangle\right|^{2}=F_{v}^{v^{\prime}}(m)\left|\left\langle\Psi_{v, 0}|\beta| \Psi_{v^{\prime}, 0}\right\rangle\right|^{2},
$$

where $F_{v}^{v^{\prime}}(m)$ is the homonymous factor. The latter takes the form of a power series,

$$
F_{v}^{v^{\prime}}=\left(1+a m+b m^{2}+\cdots\right)^{2},
$$

where $m$ is a rotational line number; in our case, $m$ takes the values $-2 J^{\prime}-3$ for $\Delta J=-2$, and $2 J+3$ for $\Delta J=2 .{ }^{25}$ Except molecular hydrogen, ${ }^{26}$ the Herman-Wallis corrections have only a very minor effect on the transitions $\Delta J=0$, so $F_{v}^{v^{\prime}}=1$. This is true irrespective of whether it is the anisotropic or the isotropic spectrum that is concerned by the branch $Q$. For the specific problem at hand, we expect that the $\Delta J= \pm 2$ transitions will display a similar behavior, so $F_{v}^{v^{\prime}} \approx 1$ for branches $O$ and $S$, also. This stems as a consequence of the fact that solely transitions involving moderate values of $J(\lesssim 20)$ were featured on the recorded spectra, while the leading-order Herman-Wallis coefficient, $a$, of $\mathrm{CO}_{2}$ typically lies in the range of $10^{-4}-10^{-3}$. The absence of any systematic deviations between calculated and recorded spectra in this range of $J$ (see below) confirms a posteriori the validity of the assumption $F_{v}^{v^{\prime}} \approx 1$.

The integrated intensity (strength) of a Raman-allowed rotational line is expressed in absolute units $\left(\mathrm{cm}^{2}\right)$ through the following equation:

$$
I_{J}=\frac{2}{15} k_{s}^{4} P_{J} b_{J}^{J^{\prime}} F_{v}^{v^{\prime}}(m)\left|\left\langle\Psi_{v, 0}|\beta| \Psi_{v^{\prime}, 0}\right\rangle\right|^{2} .
$$

Here, $k_{s}\left(=2 \pi / \lambda_{s}=2 \pi v_{s}\right)$ denotes the wave vector norm for the scattered wave, where $v_{s}=v_{L}-v_{J}$ and $v_{L}\left(=1 / \lambda_{L}\right)$ is the laser wave number. The symbol $P_{J}$ denotes the population of the initial state, i.e., the state with energy $E_{v}$ and angular momentum $J$. This population is expressed in terms of statistical weight factors $g_{J}$, as $P_{J}=g_{J}(2 J+1) Z^{-1} \exp \left(-E_{v} / k_{B} T\right)$. For the overtone band of $\mathrm{CO}_{2}, g_{J}=1$, for all the even $J$ values, but for the odd ones, it turns out that $g_{J}=0$, which means that odd- $J$ rotational lines are absent. The symbol $Z$ stands for the rotational partition function; classically, $Z=k_{B} T / 2 B$. From the elements given above, the rotational line strength of the anisotropic overtone band of $\mathrm{CO}_{2}$ reads

$$
\begin{aligned}
I_{J}= & \frac{2}{15}(2 J+1) k_{s}^{4} b_{J}^{J^{\prime}} \frac{2 B}{k_{B} T} F_{0}^{v^{\prime}}(m) \exp \left(-\frac{E_{0}}{k_{B} T}\right) \\
& \times\left|\left\langle\Psi_{0,0}|\beta| \Psi_{v^{\prime}, 0}\right\rangle\right|^{2},
\end{aligned}
$$

where $J$ is meant to be restricted to its even values only.

\section{MODELING OF LINE SHAPES}

\section{A. The hot band}

Figure 1 shows the stick spectrum of the anisotropic overtone component. In this spectrum, our simulations revealed the presence of a contribution, hitherto hidden, centered at a position close to $4648 \mathrm{~cm}^{-1}$ and identified as being due to

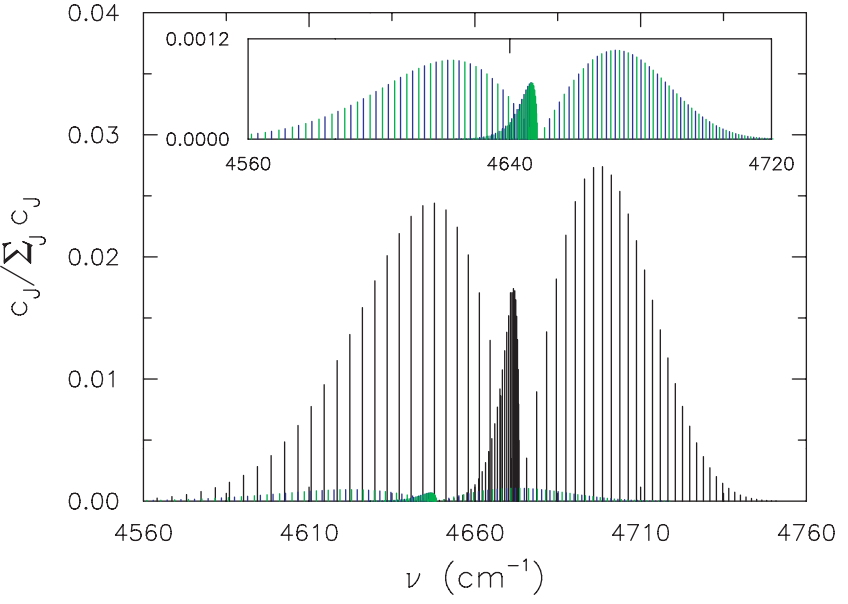

FIG. 1. Stick spectrum of the $2 v_{3}$ overtone anisotropic component. In addition to the $00^{0} 2 \leftarrow 00^{0} 0$ transition of interest, the hitherto hidden contribution of the hot transition $01^{1} 2 \leftarrow 01^{1} 0$ is now clearly visible. The horizontal axis represents Raman shift, $v$, in units of $\mathrm{cm}^{-1}$. The vertical axis represents intensity $c_{J} / \sum_{J} c_{J}$ (no units) and $c_{J}$ s are line $\delta$-function strengths. The spectrum is unit normalized. The slight prominences at the left-hand and right-hand sides of the figure as well as in its middle refer to hot band's branches $O, S$, and $Q$, respectively. A zoom into the region of the hot band is shown in the inset. The blue (dark grey) and green (light grey) sticks represent sub-band $e$ and sub-band $f$, respectively.

the hot transition $01^{1} 2 \leftarrow 01^{1} 0$. Although weak, this contribution is perceptible on the figure. A description of the hot band structure is made below in a few sentences.

Although symmetry dictates that odd $J$ lines of totally symmetrical vibrational states in ${ }^{12} \mathrm{C}^{16} \mathrm{O}_{2}$ are forbidden, the coupling between vibrational angular momentum and rotational angular momentum lifts the directional degeneracy of the former vector (with respect to the direction of the molecular axis). As a result, vibrational $l$-doubling occurs and the hot band splits up in two sub-bands. These bands are referred to as $e$ or $f$, depending on whether the rotational lines are of odd or even $J$ values, respectively.

The rotational lines of the two sub-bands have their own set of spectroscopic constants. ${ }^{23}$ These constants are gathered in Table II.

\section{B. Boltzmann statistics for $01^{1} 2 \leftarrow 01^{1} 0$ and $00^{\circ} 2 \leftarrow 00^{\circ} 0$}

The relative effects of the $01^{1} 2 \leftarrow 01^{1} 0$ and $00^{0} 2$ $\leftarrow 00^{0} 0$ transitions on the spectrum are calculated through

TABLE II. Spectroscopic constants for the $01^{1} 2 \leftarrow 01^{1} 0$ hot transition. All quantities are in $\mathrm{cm}^{-1}$.

\begin{tabular}{cccc}
\hline \hline$e$ & $v_{00}$ & $B$ & $B^{\prime}$ \\
\hline & $4648.3333031(91)$ & $0.390639109(15)$ & $0.384547434(10)$ \\
& $D \times 10^{6}$ & $D^{\prime} \times 10^{6}$ \\
& & $0.1353930(122)$ & $0.1341937(38)$ \\
& $v_{00}$ & $B$ & $B^{\prime}$ \\
& $4648.3333031(91)$ & $0.391254698(20)$ & $0.385128989(26)$ \\
& $D \times 10^{6}$ & $D^{\prime} \times 10^{6}$ \\
& $0.1361606(170)$ & $0.1361785(250)$ \\
\hline \hline
\end{tabular}


Boltzmann statistics. In this respect, the population of the hot-band ground state $01^{1} 0$, relatively to the population of the truly ground state $00^{0} 0$, reads $2 \exp \left(-v_{2} / k_{B} T\right)$. Here, the factor 2 accounts for the fact that there are two subbands; $\nu_{2}\left(=667.4 \mathrm{~cm}^{-1}\right)$ is the frequency of the bending fundamental vibration. Note that the proportion of molecules that undergo each of the two hot-doublet transitions is $\left[\exp \left(-v_{2} / k_{B} T\right)\right] /\left[1+2 \exp \left(-v_{2} / k_{B} T\right)\right]$; the proportion of molecules that undergo the transition $00^{0} 2 \leftarrow 00^{0} 0$ is $1 /\left[1+2 \exp \left(-v_{2} / k_{B} T\right)\right]$. The denominator, common to both expressions, counts the total population in the two ground states $00^{0} 0$ and $01^{1} 0$. It follows that the transition $00^{0} 2 \leftarrow$ $00^{0} 0$ contributes $92.8 \%$ of the total spectrum and that each hot sub-band makes a contribution of $3.6 \%$.

In view of the above analysis, two important questions arise and need to be answered: "Is the $2 v_{3}$ spectrum, the spectrum of the $00^{0} 2 \leftarrow 00^{\circ} 0$ transition alone?" "And if not, what does then $2 v_{3}$ truly mean?" The answer to the first question is definitely "no." As for the second one, "the $2 v_{3}$ has a spectrum, which is nothing but the combined spectrum of the two transitions $00^{0} 2 \leftarrow 00^{0} 0$ and $01^{1} 2 \leftarrow 01^{1} 0$." This important remark is in accordance with our recent study, ${ }^{16}$ in which the combined $\left(00^{0} 2 \leftarrow 00^{0} 0\right)+\left(01^{1} 2 \leftarrow 01^{1} 0\right)$ spectrum was recorded, reported, and interpreted, without explicit distinction between its main band component $00^{0} 2 \leftarrow 00^{0} 0$ and its hot band $01^{1} 2 \leftarrow 01^{1} 0$. Since the right-hand side of the formula for the vibrational matrix element $\left\langle v_{1}, v_{2}, v_{3}+2|\beta| v_{1}, v_{2}, v_{3}\right\rangle$, given in Ref. 27, does not contain the vibrational quantum numbers $v_{1}$ or $v_{2}$ [see Eq. 5 in Ref. 16], one has $\left\langle 01^{1} 2|\beta| 01^{1} 0\right\rangle$ $\approx\left\langle 00^{0} 2|\beta| 00^{0} 0\right\rangle$. Strikingly, only the combined $\left(00^{0} 2 \leftarrow 00^{0} 0\right)+\left(01^{1} 2 \leftarrow 01^{1} 0\right)$ spectrum has a spectral moment $M_{0}^{\text {ani }}$ that is temperature independent,

$$
\begin{aligned}
M_{0}^{a n i}= & \frac{1}{1+2 \exp \left(-\frac{v_{2}}{k_{B} T}\right)}\left[\left|\left\langle 00^{0} 2|\beta| 00^{0} 0\right\rangle\right|^{2}\right. \\
& \left.+2 \exp \left(-\frac{v_{2}}{k_{B} T}\right)\left|\left\langle 01^{1} 2|\beta| 01^{1} 0\right\rangle\right|^{2}\right] \\
\approx & \left|\left\langle 00^{0} 2|\beta| 00^{0} 0\right\rangle\right|^{2} .
\end{aligned}
$$

For the purposes of this study, the transition $01^{1} 2 \leftarrow 01^{1} 0$ was properly incorporated in our simulations and the spectral anomaly was completely removed.

\section{The spectrum function}

The general expression for a band spectrum function is

$$
\Phi(\omega)=\frac{1}{\pi} \operatorname{Re}\left\langle\left\langle A\left|\mathbf{R}^{-1}\right| A\right\rangle\right\rangle .
$$

In this expression, $A$ designates the irreducible spherical tensor polarizability component of rank two; for linear molecules, this component reads $\left(\alpha_{\|}-\alpha_{\perp}\right) C^{(2)}(\Omega)$, where $\beta\left(=\alpha_{\|}-\alpha_{\perp}\right)$ is the anisotropy of $\mathrm{CO}_{2}$ and $C^{(2)}(\Omega)$ the second-rank Racah harmonic. $\mathbf{R}$ is a matrix defined as $\mathbf{R}$ $=-i(\omega \mathbf{I}-\mathbf{L})+\boldsymbol{\Gamma}$, with $\boldsymbol{\Gamma}$ and $\mathbf{L}$ the relaxation matrix and the molecular Liouvillian, respectively. The latter is diagonal in the line-space basis $|m\rangle\rangle$ and obeys the eigenvalue equation $\left.\mathbf{L}|m\rangle\rangle=\omega_{m}|m\rangle\right\rangle$, where $\omega_{m}$ are the self frequencies $\omega_{f i}$ of the allowed transitions $f \leftarrow i$ in the free molecule. $\mathbf{I}$ is the identity matrix. The " $-i$ " convention used in the definition of $\mathbf{R}$ is consistent with the definition used in Forster's general treatment ${ }^{28}$ for a complex-valued response function $S(\omega)$ (see also the footnote ${ }^{29}$ ).

By inserting the unit operator $\left.\sum_{m}|m\rangle\right\rangle\langle\langle m|$ twice into Eq. (8), one obtains

$$
\Phi(\omega)=\frac{1}{\pi} \operatorname{Re} \sum_{m, m^{\prime}} A_{m} A_{m^{\prime}}\left\{[-i(\omega \mathbf{I}-\mathbf{L})+\boldsymbol{\Gamma}]^{-1}\right\}_{m, m^{\prime}} .
$$

This way to express the spectrum function is general enough to allow one to account for rotational pressure effects. These are stored in the diagonal elements of $\boldsymbol{\Gamma}$, with $\gamma_{m}$ $=\operatorname{Re} \Gamma_{m, m}$ and $\delta_{m}=\operatorname{Im} \Gamma_{m, m}$ being identified as the pressureinduced width and shift of line $m$, respectively. The integrated intensity of line $m$ is given by $A_{m}^{2}$.

Henceforward, we will assume that the spectrum is unit normalized, i.e., $\sum_{m} A_{m}^{2}=1$, and that the process is Markovian, so any frequency dependence in the relaxation matrix will be disregarded.

\section{The isolated Lorentzian model}

An isolated spectral line model was first adopted, and the rotational lines were assumed to have the same functional form and to provide additive contributions. Mathematically, this model follows from Eq. (9) upon neglecting the off-diagonal $\boldsymbol{\Gamma}$ matrix elements, so a simple spectrum function is obtained that is a sum of Lorentzians,

$$
\begin{aligned}
\Phi(\omega) & =\frac{1}{\pi} \operatorname{Re} \sum_{m} A_{m}^{2}\left[-i\left(z-\omega_{m}\right)+\Gamma_{m, m}\right]^{-1} \\
& =\frac{1}{\pi} \sum_{m} A_{m}^{2} \frac{\gamma_{m}+\gamma_{v}}{\left(\omega-\omega_{m}-\delta_{m}-\delta_{v}\right)^{2}+\left(\gamma_{m}+\gamma_{v}\right)^{2}} .
\end{aligned}
$$

Note that, here, the variable $\omega$ was continued to the complex variable $z=\omega+i\left(\gamma_{v}+i \delta_{v}\right)$, where the parameter $\gamma_{v}+i \delta_{v}$ was used to ad hoc describe vibrational pressure broadening and shift. ${ }^{29}$ The latter constants, along with the rotational pressure broadening and shift, form what in line shape theory is termed "the pressure-induced coefficients." In this paragraph, we make the assumption that the line shifts $\delta_{m}$ (which are usually much smaller than the line widths $\gamma_{m}$ ) were negligible. This model will be referred to as A.

Figure 2 shows the theoretical spectrum, as it was calculated with model A, in comparison with the experimental spectrum. The spectra are unit normalized. The abscissa represents Raman frequency shift (in $\mathrm{cm}^{-1}$ units). The gas density is 5 amagat. The excellent agreement between the two spectra, at this density, is evidence of the adequacy (at least as seen with the naked eye) of model A.

On increasing the gas density, the situation is seen to change dramatically. Figure 3 illustrates the two spectra at a 


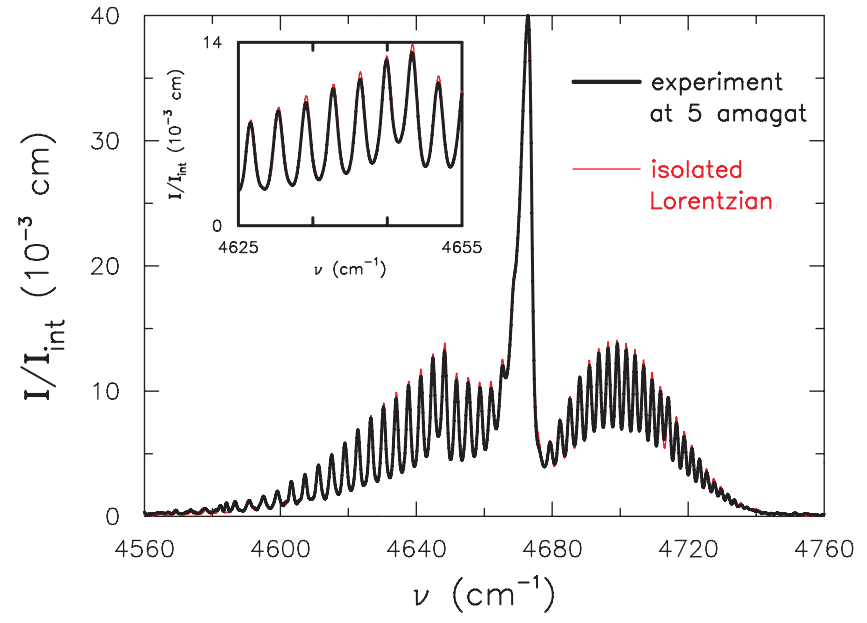

FIG. 2. Calculated [thin red (thin light grey) line] and recorded [thick black (thick dark grey) line] spectrum intensities $I / I_{\text {int }}\left(=I / \int I d v\right)$, in $\mathrm{cm}$ units, as a function of Raman shift, $v$, in units of $\mathrm{cm}^{-1}$. Model A was employed for the calculations. The gas temperature is $294.5 \mathrm{~K}$. The gas density is 5 amagat. Both spectra are unit normalized. Inset: zoom into the region of the anomaly to better appreciate the quality of the simulation.

density of 30 amagat [panel (a)], and then at 55 amagat [panel (b)]. The strong overlap between rotational lines makes the rotational structure of Fig. 2 completely disappear from Fig. 3. In both figures the widths of the Lorentzians were assumed to scale linearly with the gas density. While the recorded band shape is still reproduced qualitatively, an excess intensity is progressively accumulated in the wings of the $S$ and $O$ branches at the expense of the central region of the $Q$ branch. At the higher gas density, the disagreement between theoretical and experimental shapes becomes striking. This observation provides evidence for the inadequacy of model A.

In a more realistic simulation, the latter model should be replaced by a more elaborate one, in order to account for the substantial line mixing and the thereby generated intensity transfer. Let us briefly discuss the two most important such models.

\section{E. The strong-collision model}

Among the various steps that marked the development of band shape modeling, the quantum strong-collision model (SCM) (and its by-products) (Ref. 30) is probably the most important one.

The SCM's main assumption is that

$$
\Gamma_{m, m^{\prime}}=\Gamma\left(\delta_{m, m^{\prime}}-A_{m} A_{m^{\prime}}\right) .
$$

Since $A_{m}^{2} \ll 1$, all the rotational lines within this model have approximately the same width, $\Gamma_{m, m} \approx \Gamma$. The parameter $\Gamma$ denotes the effective collision rate. The assumption of Eq. (11) is in accordance (see Appendix, Part I) with two conditions for the relaxation matrix that arise in performing matrix operations in the line space. These conditions are fundamental to the correct description of a band shape because they are responsible for the transfer of spectral intensity from the edge of the band to its center. They are called "sum rules"
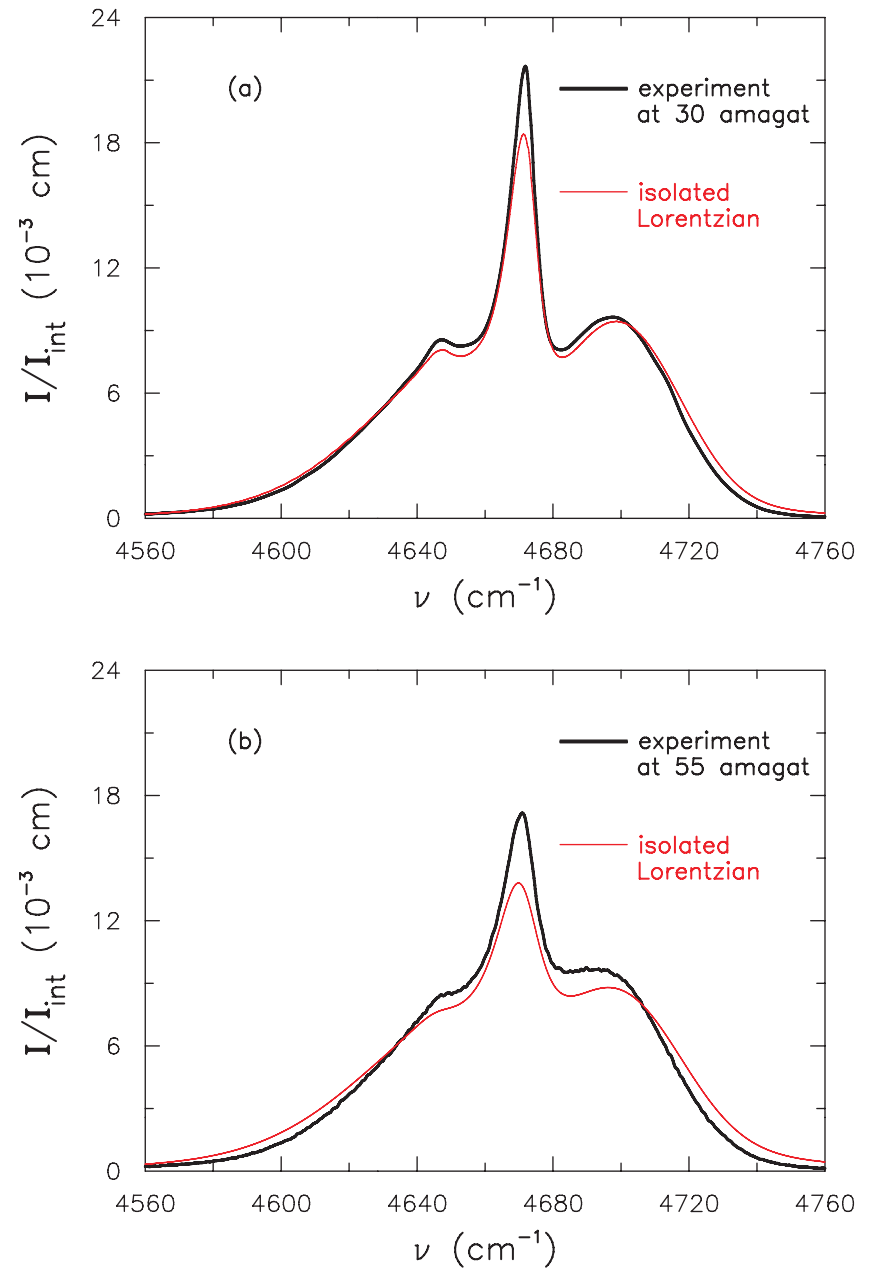

FIG. 3. Calculated [thin red (thin light grey) line] and recorded [thick black (thick dark grey) line] spectrum intensities $I / I_{\text {int }}\left(=I / \int I d v\right)$, in cm units, as a function of Raman shift, $v$, in units of $\mathrm{cm}^{-1}$. Model A was employed for the calculations. The gas temperature is $294.5 \mathrm{~K}$. The gas density is: 30 amagat [panel (a)]; 55 amagat [panel (b)]. All spectra are unit normalized.

and $\operatorname{read}^{31}$

$$
\sum_{m^{\prime}} A_{m^{\prime}} \Gamma_{m, m^{\prime}}=\sum_{m} A_{m} \Gamma_{m, m^{\prime}}=0 .
$$

It follows from the sum rules that the spectral band gets narrowed as the frequency of the collisions is increased. At higher gas densities, the line structure coalesces and a quasiLorentzian line tends to emerge as the result of line mixing. Analogous to Dicke narrowing, this line is getting sharper with increasing gas density. This is known as the motional narrowing effect.

The SCM's main advantage is that the inversion $\mathbf{R}^{-1}$ [see Eq. (8)] can be done analytically. Therefore, construct a task to calculate a band shape can be as simple as to calculate $\Phi(\omega)$ with a diagonal relaxation matrix. Moreover, this model gives a reasonable approximation overall to coalesced band spectra, especially for heavy linear molecules. The most significant shortcoming with the SCM is that it fails to describe isolated line profiles, since it predicts widths that are practically the same for all the lines. This is by no means true at low gas pressures. 
How to improve the SCM in the low pressure regime is treated in the next paragraph.

\section{F. The extended strong-collision model}

In the effort to improve the SCM, an ESCM was developed, ${ }^{17}$ which still retains all the advantages of the SCM. Among the various problems of spectroscopy that were solved with the ESCM, the interpretation of the isotropic CARS spectrum of the $\mathrm{CO}_{2}$ hot transition $11^{1} 0 \leftarrow 01^{1} 0$ is probably the most challenging one. ${ }^{11}$ This model will be referred to henceforward as B.

By contrast with Eq. (11), here, the relaxation matrix reads

$$
\Gamma_{m, m^{\prime}}=\gamma_{m}^{\prime}\left(\delta_{m, m^{\prime}}-\frac{\gamma_{m^{\prime}}^{\prime}}{\left\langle\gamma^{\prime}\right\rangle} A_{m} A_{m^{\prime}}\right) .
$$

The primed quantities $\gamma_{m}^{\prime}$ are effective line widths appearing as diagonal elements of a matrix $\boldsymbol{\gamma}^{\prime}$. The latter is a diagonal matrix, $\gamma_{m, m}^{\prime} \equiv \gamma_{m}^{\prime}$, defining the spectral shape. The appearance of $\left\langle\gamma^{\prime}\right\rangle$ here is to ensure the fulfillment (see Appendix, Part II) of the sum rules of Eq. (12). This quantity has the meaning of some average line broadening over the band and reads $\left\langle\gamma^{\prime}\right\rangle=\sum_{m} A_{m}^{2} \gamma_{m}^{\prime}$.

Since for heavy molecules line intensity amplitudes are small $\left(A_{m}^{2} \ll 1\right)$, there is only a little difference between the (primed) effective quantities $\gamma_{m}^{\prime}$ and the (unprimed) measured halfwidths $\gamma_{m}\left[=\Gamma_{m, m}=\gamma_{m}^{\prime}\left(1-A_{m}^{2} \gamma_{m}^{\prime} /\left\langle\gamma^{\prime}\right\rangle\right)\right]$, so a few iterations in Eq. (13) suffice for convergence. The thereby obtained set of $\gamma_{m}^{\prime}$ 's permits the exact description of the band at a low gas pressure.

Thanks to the simple single-term form of the off-diagonal part of $\boldsymbol{\Gamma}$, significant simplifications in the expression of the spectrum function are possible. In this respect, one of us has worked out the following analytic expression: ${ }^{18}$

$$
\Phi(\omega)=\frac{1}{\pi} \operatorname{Re}\left[\left\langle\left\langle A\left|\mathbf{R}_{d}^{-1}\right| A\right\rangle\right\rangle+\frac{\left\langle\left\langle A\left|\mathbf{R}_{d}^{-1} \boldsymbol{\gamma}^{\prime}\right| A\right\rangle\right\rangle^{2}}{\left\langle\gamma^{\prime}\right\rangle-\left\langle\left\langle A\left|\mathbf{R}_{d}^{-1} \boldsymbol{\gamma}^{\prime 2}\right| A\right\rangle\right\rangle}\right] .
$$

In this expression, $\mathbf{R}_{d}=-i(z \mathbf{I}-\mathbf{L})+\boldsymbol{\gamma}^{\prime}$, so $\mathbf{R}_{d}$ is diagonal. This property, along with the expression for $\mathbf{R}_{d}$, allows one to derive simple and general analytic formulas for the spectral sums $\left\langle\left\langle A\left|\mathbf{R}_{d}^{-1} \boldsymbol{\gamma}^{\prime n}\right| A\right\rangle\right\rangle(n=0,1,2)$. These formulas read

$$
\left\langle\left\langle A\left|\mathbf{R}_{d}^{-1} \boldsymbol{\gamma}^{\prime n}\right| A\right\rangle\right\rangle=\sum_{m} A_{m}^{2} \frac{\gamma_{m}^{\prime n}}{-i\left(z-\omega_{m}\right)+\gamma_{m}^{\prime}} .
$$

As before with model $\mathrm{A}$, here again $z=\omega+i\left(\gamma_{v}+i \delta_{v}\right)$, with the parameter $\gamma_{v}+i \delta_{v}$ intended to account for vibrational pressure effects through adjustment of the model shapes to the experiment.

The expression of Eq. (14) was used to independently simulate the (unit normalized) shapes of the $00^{0} 2 \leftarrow 00^{\circ} 0$ and $01^{1} 2 \leftarrow 01^{1} 0$ bands. The computed spectra were then weighted according to their relative initial-state populations and summed to form the overtone spectrum. To this purpose, a FORTRAN code was developed. As input values for the rotational broadening coefficients, infrared spectroscopic data ${ }^{19}$

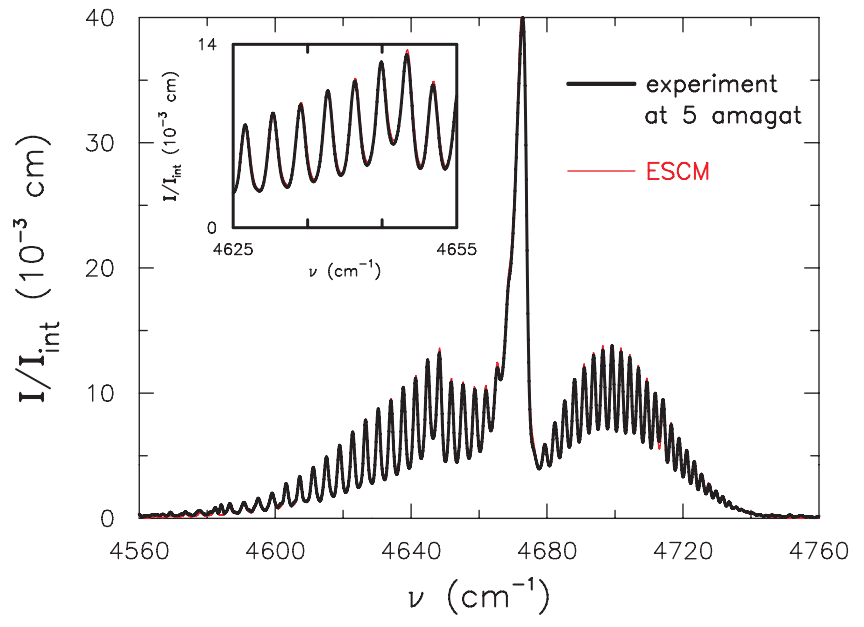

FIG. 4. Calculated [thin red (thin light grey) line] and recorded [thick black (thick dark grey) line] spectrum intensities $I / I_{\text {int }}\left(=I / \int I d v\right)$, in cm units, as a function of Raman shift, $v$, in units of $\mathrm{cm}^{-1}$. Model B (ESCM) was employed for the calculations. The gas temperature is $294.5 \mathrm{~K}$. The gas density is 5 amagat. Both spectra are unit normalized. Inset: zoom into the region of the anomaly to better appreciate the quality of the simulation.

were taken. A $0.7 \mathrm{~cm}^{-1}$ FWHM triangular function was used to mimic the slit function of the experiment.

Figure 4 shows the ESCM spectrum along with the experimental one, as a function of Raman frequency shift (in $\mathrm{cm}^{-1}$ units). The gas density is 5 amagat. As before, the intensity is unit normalized. The theoretical spectrum is hardly distinguishable from the recorded one.

Figure 5 illustrates the ESCM spectrum along with the recorded one for a gas density $\rho=30$ amagat [Fig. 5(a)] and 55 amagat [Fig. 5(b)]. The excellent response of the ESCM is clear from the figures. This performance was seen to persist at all the gas densities. By comparison with the response of model A (see Fig. 3), this is evidence of a major improvement in the band shape modeling.

Our calculations showed that vibrational broadening had too weak an effect to sizably change the simulated line shapes. It is a rather general feature of the anisotropic spectra to exhibit values of $\gamma_{v}$ that are much smaller than (and completely masked by) the line widths $\gamma_{m}$. This feature hindered the observation of vibrational broadening in our analysis. This is by no means the case with the vibrational shift, $\delta_{v}$. As mentioned earlier in this article, rotational line shifts $\delta_{m}\left(\ll \gamma_{m}\right)$ are negligible, so they do not mask the presence of $\delta_{v}$. Accurate $\delta_{v}$ values were extracted from the recorded spectra, by using model $\mathrm{B}$, which were found to scale linearly with the gas density as

$$
\delta_{v}\left(\mathrm{~cm}^{-1}\right)=-34(3) \times 10^{-3} \rho(\text { amagat }) .
$$

It is remarkable that the value 0.034(3) given above is three and six times greater than the values $-\delta_{v} / \rho \approx 0.01$ and $0.005 \mathrm{~cm}^{-1}$ /amagat obtained for the Raman allowed low and high frequency components of the $\mathrm{CO}_{2}$ doublet $v_{1}: 2 v_{2}^{0}$ Fermi resonance, respectively. ${ }^{7,11}$

\section{SYNOPSIS}

We reported a line shape analysis of the anisotropic Raman $2 v_{3}$ overtone spectrum of $\mathrm{CO}_{2}$ at room temperature 

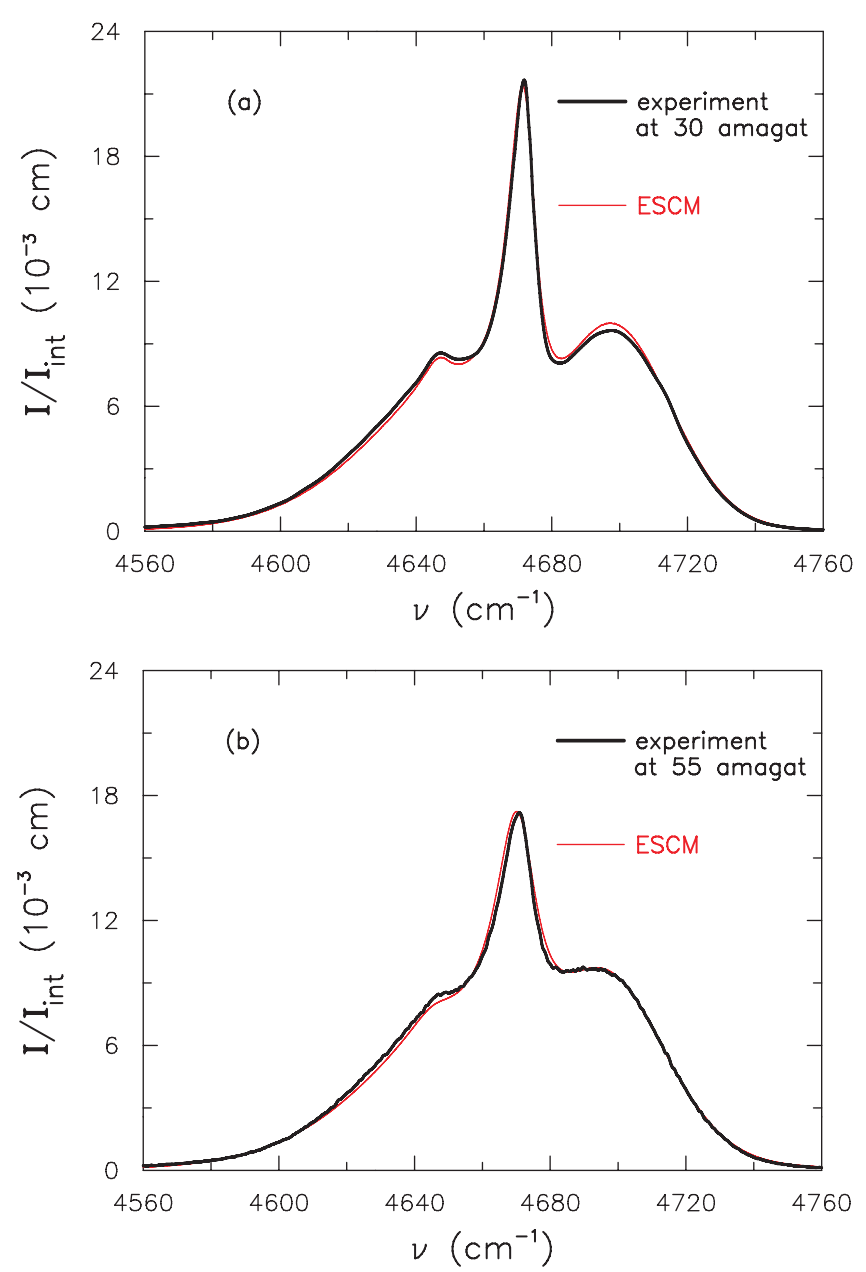

FIG. 5. Calculated [thin red (thin light grey) line] and recorded [thick black (thick dark grey) line] spectrum intensities $I / I_{\text {int }}\left(=I / \int I d v\right)$, in $\mathrm{cm}$ units, as a function of Raman shift, $v$, in units of $\mathrm{cm}^{-1}$. Model B (ESCM) was employed for the calculations. The gas temperature is $294.5 \mathrm{~K}$. The gas density is: 30 amagat [panel (a)]; 55 amagat [panel (b)]. All spectra are unit normalized.

and for a range of gas densities varying from 5 to 55 amagat. The calculation of the spectrum was based mainly on the ESCM, which is a semi-analytical quantum mechanical model. Striking agreement at any density was observed between the ESCM spectra and the experiment, which permitted the successful interpretation of the experiment and a better understanding of the substantial line mixing effects. The presence of a hot band affecting the spectrum by about $7 \%$ was revealed. Calculated input data for the rotational broadening coefficients were employed, which were then used to derive the vibrational shift of the band by globally adjusting the ESCM spectra to the experiment.

Our work appears as the last word on an issue as timely as the advanced line shape theory of carbon dioxide. The ESCM adopted here appears as the most viable compromise between performance and effort. The elegance of its formalism and its easy numerical implementation are also aspects to stress.

\section{APPENDIX: SUM RULES IN THE SCM AND ESCM}

\section{Part I}

The SCM assumes that $\Gamma_{m, m^{\prime}}=\Gamma\left(\delta_{m, m^{\prime}}-A_{m} A_{m^{\prime}}\right)$. In order to check the validity of the sum rules under this assumption, let us calculate the expressions (i) $\sum_{m^{\prime}} A_{m^{\prime}} \Gamma_{m, m^{\prime}}$ and (ii) $\sum_{m} A_{m} \Gamma_{m, m^{\prime}}$. For (i) we find

$$
\begin{aligned}
\sum_{m^{\prime}} A_{m^{\prime}} \Gamma_{m, m^{\prime}}= & \Gamma \sum_{m^{\prime}} A_{m^{\prime}}\left(\delta_{m, m^{\prime}}-A_{m} A_{m^{\prime}}\right) \\
= & \Gamma\left[\sum_{m^{\prime} \neq m} A_{m^{\prime}}\left(\delta_{m, m^{\prime}}-A_{m} A_{m^{\prime}}\right)\right. \\
& \left.+A_{m}\left(1-A_{m}^{2}\right)\right] \\
= & \Gamma A_{m}\left[-\left(\sum_{m^{\prime} \neq m} A_{m^{\prime}}^{2}\right)+\left(1-A_{m}^{2}\right)\right] \\
= & \Gamma A_{m}\left[1-\sum_{m^{\prime}} A_{m^{\prime}}^{2}\right]=0 ;
\end{aligned}
$$

likewise for (ii).

\section{Part II}

In the ECSM, the assumption is $\Gamma_{m, m^{\prime}}=\gamma_{m}\left[\delta_{m, m^{\prime}}\right.$ $\left.-\left(\gamma_{m^{\prime}} /\langle\gamma\rangle\right) A_{m} A_{m^{\prime}}\right]$. We will check the validity of the two sum rules (i) $\sum_{m^{\prime}} A_{m^{\prime}} \Gamma_{m, m^{\prime}}$ and (ii) $\sum_{m} A_{m} \Gamma_{m, m^{\prime}}$ under this assumption. For (i) we find,

$$
\begin{aligned}
\sum_{m^{\prime}} A_{m^{\prime}} \Gamma_{m, m^{\prime}}= & \gamma_{m} \sum_{m^{\prime}} A_{m^{\prime}}\left(\delta_{m, m^{\prime}}-\frac{\gamma_{m^{\prime}}}{\langle\gamma\rangle} A_{m} A_{m^{\prime}}\right) \\
= & \gamma_{m}\left[\sum_{m^{\prime} \neq m} A_{m^{\prime}}\left(\delta_{m, m^{\prime}}-\frac{\gamma_{m^{\prime}}}{\langle\gamma\rangle} A_{m} A_{m^{\prime}}\right)\right. \\
& \left.+A_{m}\left(1-\frac{\gamma_{m}}{\langle\gamma\rangle} A_{m}^{2}\right)\right] \\
= & \gamma_{m} A_{m}\left[-\left(\sum_{m^{\prime} \neq m} \frac{\gamma_{m^{\prime}}}{\langle\gamma\rangle} A_{m^{\prime}}^{2}\right)+\left(1-\frac{\gamma_{m}}{\langle\gamma\rangle} A_{m}^{2}\right)\right] \\
= & \gamma_{m} A_{m}\left(1-\frac{1}{\langle\gamma\rangle} \sum_{m^{\prime}} \gamma_{m^{\prime}} A_{m^{\prime}}^{2}\right)=0, \quad \text { (A2) }
\end{aligned}
$$

where use of the definition $\langle\gamma\rangle=\sum_{m^{\prime}} \gamma_{m^{\prime}} A_{m^{\prime}}^{2}$ was made; likewise for (ii).

${ }^{1}$ P. W. Anderson, Phys. Rev. 76, 647 (1949).

${ }^{2}$ M. Baranger, Phys. Rev. 111, 481 (1958); ibid. 112, 855 (1958).

${ }^{3}$ A. C. Kolb and H. Griem, Phys. Rev. 111, 514 (1958).

${ }^{4}$ U. Fano, Phys. Rev. 131, 259 (1963).

${ }^{5}$ A. Ben-Reuven, Phys. Rev. 145, 7 (1966).

${ }^{6}$ J. Bonamy, L. Bonamy, and D. Robert, J. Chem. Phys. 67, 4441 (1977).

${ }^{7}$ R. B. Wright and C. H. Wang, J. Chem. Phys. 58, 2893 (1973).

${ }^{8}$ C. M. Roland and W. A. Steele, J. Chem. Phys. 74, 2733 (1981).

${ }^{9}$ J. Baran, A. Grofcsik, and W. J. Jones, Mol. Phys. 45, 1291 (1982). 
${ }^{10}$ B. Lavorel, G. Millot, R. Saint-Loup, H. Berger, L. Bonamy, J. Bonamy, and D. Robert, J. Chem. Phys. 93, 2176 (1990).

${ }^{11}$ A. P. Kouzov, D. N. Kozlov, and B. Hemmerling, Chem. Phys. 236, 15 (1998).

${ }^{12}$ A. Predoi-Cross, W. Liu, C. Holladay, A. V. Unni, I. Schofield, A. R. W. McKellar, and D. Hurtmans, J. Mol. Spectrosc. 246, 98 (2007).

${ }^{13}$ L. Frommhold, Adv. Chem. Phys. 46, 1 (1981).

${ }^{14}$ Collision- and Interaction-Induced Spectroscopy, edited by G. C. Tabisz and M. N. Neuman (Kluwer, Dordrecht, 1995).

${ }^{15}$ I. A. Verzhbitskiy, M. Chrysos, F. Rachet, and A. P. Kouzov, Phys. Rev. A 81, 012702 (2010); M. Chrysos and I. A. Verzhbitskiy, ibid. 81, 042705 (2010); I. A. Verzhbitskiy, M. Chrysos, and A. P. Kouzov, ibid. 82, 052701 (2010).

${ }^{16}$ M. Chrysos, I. A. Verzhbitskiy, F. Rachet, and A. P. Kouzov, J. Chem. Phys. 134, 044318 (2011).

${ }^{17}$ J. P. Sala, J. Bonamy, D. Robert, B. Lavorel, G. Millot, and H. Berger, Chem. Phys. 106, 427 (1986).

${ }^{18}$ A. P. Kouzov, Chem. Phys. Lett. 188, 25 (1992).

${ }^{19}$ L. Rosenmann, J.-M. Hartmann, M. Y. Perrin, and J. Taine, Appl. Opt. 27, 3902 (1988)

${ }^{20}$ F. Rachet, Y. Le Duff, C. Guillot-Noël, and M. Chrysos, Phys. Rev. A 61, 062501 (2000).

${ }^{21}$ P. Atkins and J. de Paula, Atkins' Physical Chemistry (Oxford University Press, London, 2006).
${ }^{22}$ G. Herzberg, Molecular Spectra and Molecular Structure. Part II. Infrared and Raman Spectra of Polyatomic Molecules (Van Nostrand, Princeton, 1966).

${ }^{23}$ C. E. Miller and L. R. Brown, J. Mol. Spectrosc. 228, 329 (2004).

${ }^{24}$ R. Herman and R. F. Wallis, J. Chem. Phys. 23, 637 (1955).

${ }^{25}$ M. A. Buldakov, V. N. Cherepanov, B. V. Korolev, and I. I. Matrosov, J. Mol. Spectrosc. 217, 1 (2003).

${ }^{26}$ M. Marrocco, Chem. Phys. Lett. 442, 224 (2007).

${ }^{27}$ T. D. Kolomiitsova and D. N. Shchepkin, in Advances in Spectroscopy, edited by R. J. H. Clark and R. E. Hester (Wiley, Chichester, 1995), Vol. 23.

${ }^{28}$ D. Forster, Hydrodynamic Fluctuations, Broken Symmetry, and Correlation Functions (Benjamin, Reading, MA, 1975).

${ }^{29}$ In order to obtain the physical band spectrum, the time correlation function $\langle\langle A(t) \mid A(0)\rangle\rangle$ had to be Laplace-transformed with $\exp (i z t)$, where $z=\omega+i \varepsilon(\varepsilon \rightarrow 0)$, and $\varepsilon>0$, so that convergence is ensured. Yet, upon formulating the method to ad hoc incorporate vibrational relaxation by means of $\gamma_{v}+i \delta_{v}$, the parameter $\varepsilon$ becomes redundant, since the convergence of the transform is now ensured by the real part of $\gamma_{v}+i \delta_{v}$, which is positive. As for the "plus" sign in the imaginary part, this sign is in accordance with the conventional definition of a lineshift.

${ }^{30}$ A. I. Burshtein and S. I. Temkin, Spectroscopy of Molecular Rotation in Gases and Liquids (Cambridge University Press, Cambridge, England, 1994).

${ }^{31}$ N. N. Filippov and M. V. Tonkov, J. Chem. Phys. 108, 3608 (1998). 